\title{
Paul du Bois-Reymond.
}

In Paul du Bois-Reymond betrauern wir einen Manu, der, wie ex in der Wissenschaft durch seine Forschungen einen bleibenden Xinen sich exworben, so auch in der Erinnerung der Freunde durch die scharfgeschnittenen Züge einer eigenartigen Persönlichkeit ein bleibrndes Bild und eine fühlbare Lücke zurückgelassen hat.

Die glänzenden Gaben des Geistes, welche ihm die Natur verlielien l,atte, wurden entfaltet und entwickelt unter dem befruchtenden Einins günstiger Lmstände and eines reichen Lebens. Paul du Bois-Reymond wurde geboren am 2. Dezember 1831 in Berlin als Sohn des dort angesessenen Neuenburgers Felix Henri du Bois-Reymoud, der als socalpolitischer Schriftsteller, als Sprachforscher und höherer Verwaltungsbeamter einen geachteten Namen und angesehene Stellung besass.

Die Nutter entstammte der französischen Colonie in Burlin un? dem entsprechend war die Sprache des elterlichen Hauses und dic Erziehung, die Paul mit seinem älteren Bruder Enil, lem berihmton Physiologen, gemeinsam hatte, vorwiegend französinch. Die Söhn sprachen mit dem Vater nie ein deutsches Wort. Nit Licbe lat Paul stets an den Erinnerungen des Vaterhauses gehangen und das Verhaltniss unter den Geschwistern war ein sehr inuiges.

An sein romanisches Blut, die Einflüsse des franzönischen Elementes in seiner Jugend, das sich wit einer gut deutschen (jesinung woh vertrug, mahnten manche Züge seives Wesens, die Lebhaftigkeit sein's Temperamentes, die Vielseitigkeit und Gewandtheit seiner Conversation, die Fülle treffender Bilder, die ex im müudlichen und schrfflichen Ausdruck einzuflechten liebte.

Seine Vorbildung erhielt Paul da Bois:Reymond auf dem französischen Gymnasium in Berlin, dem Collére in Nenchatel, nand dom Gymnasium zu Naumburg. Sein Universitätsstadium, welches zniächst der Medicin galt, begann er in Zürich (1853). Schon danals lat er mit seinen Freande Adolf Fick gemeinsam tine ledeutende lintr- 
suchung iber den blinden Fleck im menschlichen Auge angestellt und veröffentlicht.

Allein er blieb nicht bei der Medicin; seine Neigung für die Mathematik war unwiderstehlich. Sie trieb ihn nach Königsberg, wo damals Eranz Neumann in der Blüthe seiner Thätigkeit stand und daneben Richelot das mathematische Studium mit der ihm eigenen Begeisterung und Hingabe leitete.

Hier wurde du Bois-Reymond zunächst auf die mathematische Physik geführt, wobei ihn seine ansgezeichnete Beobachtungsgabe, die auch den alltäglichsten Erscheinungen eine interessante und lehrreiche Seite abzugewinnen wusste, unterstützte. Und wenn er auch später der reinen Mathematik sich zuwandte, so wurden doch durch den Ausgangspunkt von der mathematischen Physik Richtung und Ziele seiner späteren Forschungen wesentlich bestimmt.

Im Jahre 1859 promovirte er in Berlin mit einer Dissertation "de aequilibrio fluidorum" nachdem bereits mehrere die Kapillaritätserscheinungen betreffende theils theoretische theils experimentelle Untersuchungen vorhergegangen waren.

Mehrere Jahre wirkte er nun als Oberlehrer der Mathematik und Physik am Friedrich-Werder'schen Gymnasium in Berlin, eifrig beschäftigt mit mathematischen Studien, denen als erste Frucht seine "Beiträge zur Interpretation der partiellen Differentialgleichungen mit drei Variablen" entsprang, ein bedeutendes Werk, das gewissermassen das Programm seiner späteren Forschungen enthält, und Zeugniss eines hervorragenden Talentes ist, welches fern von jedem $\mathrm{Zwang}$ eiver mathematischen Schule und unbeirrt durch hergebrachte Meinungen seine eigenen Wege geht. Anknüpfend an die von Monge geschaffene Theorie der Charakteristiken, die er in hohem Masse fruchtbar zu machen weiss, geht der Verfasser in dieser Schrift weniger darauf aus, die Integration der partiellen Differentialgleichungen in einzelnen Fällen zu lehren, als vielmehr den Inhalt und die Bedeutung einer partiellen Differentialgleichung und ihrer Integrale geometrisch anschaulich synthetisch zu entwickeln.

Im Jahre 1865 siedelte P. du Bois-Reymond nach Heidelberg über, um sich der akademischen Laufbahn zu widmen. Es war damals noch die goldene Zeit der Naturwissenschaften an der Heidelberger Universität, wo der gefeierte Name Bunsens die Chemiker aus der ganzen Welt heranzog, wo die Entdeckung der Spectralanalyse durch Kirchhoff und Bunsen noch in frischer Erinnerung lebte, wo Helmholtz als Lehrer der Physiologie und als Forseher anf dem Gebiete der mathematischen Physik glänzte und Hesse durch seine analytische Geometrie Sinn für elegante Form in mathematischen Untersuchungen unter seinen Schülern wechte. 
Hier trat Paul du Bois-Reymond in einen wïssenschaftlich angeregten Kreis und war, da er selbst viel beizustenern batte, überall gern aufgenommen. In jener Zeit war es auch, wo der Schreiber dieser Zeilen mit ihm bekannt wurde, und wo sich eine Freundschaft anknüpfte, die auch nach der räumlichen Trenoung Stand hielt, bis der Tod sie löste.

Paul du Bois-Reymond war eine durchaus ursprüngliche Natur, die sich nicht zu verstellep, nicht zurückzuhalten wusste, sich immer gab, wie sie war und empfaud. Während ihm nichts ferner lag, nichts unsympathischer war, als einem Modeinteresse nachzugehen, hatte er ein warmes Gemüth für die Natur, er liebte die Musik und liess selten eine Gelegenheit ungenutzt vorübergehen, eine Mozart'sche Oper oder eine Beethoven'sche Symphonie mit anzuhören. In den seinem Fache verwandten Wissenschaften batte er ein eindringendes Verständniss und ein scharfes und treffendes Urtheil über Werth oder Unwerth einer neuen Erscheinung.

Im Mittelpupkt seiner Interessen stand aber imner und jederzeit seine eigene Wissenschaft die Mathematik, und keine Unterhaltung war ihm willkommener als ein Gespräch mit einem Fachgenossen über die ihn bewegenden wissenschaftlichen Fragen. Er war ein stets aufgelegter fröhlicher Geselle beim Glase oder bei Ausliügen, die er sehr liebte und durch seine treffenden Beobachtungen und seinen guten Humor zu beleben wusste; er konnte aber auch rücksichtslos scharf und verletzend sein, wo ef sich gekränlt glaubte oder wo er auf Anschauungen stiess, die ihm verwerflich erschienen. Solche Anlïsse boten sich mannigfach in den aufgeregten Tagen des Jahres 1866, wa er mit seinem ganzen Herzen auf der Seite Preussens stand.

Im Verkehr mit seinen Zuhörern, deren er schon als Privatdocent einen ansehnlichen $K_{r}$ eis um sqch versammelte, denen er auch ausser der Zeit der Vorlesungen manche Stunde widmete, wirlte er sehr an" regend, wie die zablreichen aus seiner Schule hervorgegangenen grösseren und kleineren Publicationen beweisen, und mancher seiner Schüler hat sich bereits einen geachteten Namen als Lehrer an einer Hochschule and als wissenschaftlicher Forscher erworben.

Die ersten Arbeiten, die du Bois-Reymond in seiner Heidelberiwer Zeit veröffentlichte, bezogen sich noch auf die partiellen Differentialgleichungen. Bald jedoch führten ihn diese Studien auf die genauere Untersuchung des wichtigsten Hilfsmittels bei der Integration der partiellen Differentialgleichungep, der Fourier'schen Reihen und Integrale und der verwandten Sätze, die er unter dem Namen der "Darstellungsformeln" zusammengefasst hat. Dieser Gegenstand hat seine Arbeitskraft am längsten beschäftigt, und dieser /weig der Analysis verdankt ihm wesentijeke Fortschritte. 
Die Theorie der Fourier'schen Reihen war durch die berühmte Dirichlet'sehe Untersuchung (im $4^{\text {ten }}$ Band des Crelle'schen Journals) ¿war fär eine grosse Classe von Functionen vollendet, sie forderte aber nach zwei Seiten hin eine Weiterfübrung und Verallgemeinerung; einmal handelte es sich um die Frage, wie man andere allgemeinere Functionen als die trigonometrischen zu analogen Darstellungen benutzen könnte, andererseits darum, wie der Kreis der Functionen abzugrenzen sei, welche eine solche Entwicklung zulassen. Dirichlet hatte bei seiner Untersuchung solche Functionen ausgeschlossen, welche nnendlich viele Maxima und Minima haben, war aber, wie es scheint, des Glaubens, dass auch auf diese Functionen and besonders auf alle stetigen Functionen die Fourier'schen Entwicklungen anwendbar seien. Arbeiten von Lipschitz and Riemann hatten die Untersuchung von Dirichlet zwar weitergefübrt und manches Neue binzugefügt; aber der Beweis für die Entwickelbarkeit aller stetigen Functionen stand immer noch aus als du Bois-Reymond den Gegenstar à aufnahm. Nachdem alle Versuche, einen strengen Beweis zu fuden, fehlgeschlagen waren, gelangt er erst zu dem Zweifel an der Richtigkeit und endlich zum Beweis der Unrichtigkeit, indem er eine zwar stetige aber mit unendlich vielen Maximis und Minimis behaftete Function bildet und discutirt, für welche er die Divergenz der Fourier'schen Entwicklung nachweisen kann.

So ist also diese Frage zu einem dem erwarteten und gesuchten ganz entgegengesetzten Abschluss gebracht und weun auch damit das letzte Ziel, nothwendige und hinreichende Bedingungen für die Entwickelbarkeit einer Function zu finden, welches du Bois-Reymond äberhaupt für unerrejchbar hielt, nicht gewonven ist, so ist doch zum erstenmal, wenn der Ausdruck gestattet ist, eine obere Grenze für den Bereich der entwickelbaren Functionen gegeben.

Die Resultate dieser Untersuchnngen über die Fourier'schen Darstellungsformeln sind in mehreren Abhandlungen, die theils im Crelle'scben Journal, theils in diesen Annalen und in anderen Zeitschriften erschienen sind, am Ausführlichsten und im Zusammenhang in einer in den Schriften der Münchner Akademie rom Jahre 1876 abgedruckten Abhandlung dargestellt.

Das wichtigste Hilfsmittel dieser Untersuchungen war ihm der Mittelwerthsatz fïr bestimmte Integrale, in dessen Besitz er sehon frühzeitig war, der, wenn er auch von Weierstrass bereits gekannt und in Vorlesungen erwähnt war, doch von du Bois-Reymond selbständig gefunden und zuerst in voller Allgemeinheit bewiesen wurde, und daher mit Recht unter dem Namen des du Bois-Reymond'seben Mittelwerthsatzes bekannt ist, ein Satz der bei der Untersuchung bestimmter Integrale, namentlich wenn es sich um die Frage nach der Umkehrbarkeit vielfacher Integrale handelt, von grösstem Nutzen ist. 
Durch diese Untersuchangen wurde du Bois-Reymond mehr und meler auf die fundamentalen Fragen der reellen Functionentheorie geführt, wobei ihm seine Beziehungen zu Weierstrass, die er sehr hoch sehätzte und durch häufige Besuche in Berlin zu pflegen suchte, selir förderlich waren. In den sechziger Jahren warde, angeregt durch Riemann and Weierstrass, die Differentiirbarkeit und die Mögliohkeit stetiger Functionen ohne Differentialquotienten lebhaft unter den Mathematikern erörtert. Du Bois-Reymond gehörte zu denjenigen, welche lange an der Existenz solcher Functionen 2weifelten; seine Arbeitsrichtung wies ibn aber mit Nothwendigkeit auf eine Entscheidung dieser Frage hin, und das ron ihm selbst veröffentlichte Beispiel, auf welches ihn Weierstrass hingewiesen hatte (Versuch einer Classification der willkürlichen Functionen reeller Argumente nach ihren Aenderungen ii deu bleinsten Iutervallen, (Journal f. Jathem. Bd.79) mqisste schliesslich den bartnäckigsten $\mathrm{Zweifler}$ uberzeugen, wenn er sich nur die Mähe nahm, dem einfachen Bepeise Schritt für Schritt zu folgen.

Jahrelang trug sich du Bois-Reymond, wie er selbst in einer iul Jahre 1878 an die Fachgenossen versandten Anlündigung berichtet, mit dem Gedanken, die Ergeknisse seiner Forschungen systematisch darzustellen, ausgehend rou einer philosophischen Erörterung der Grundbegriffe der Zahl, der Stetigkeit und der Grenze. Der im Jahre 1882 erschienene erste Theil der "allgemeinen Funptionentheorie", „Metaphysik und Theorie der mathematischen Grundbegriffe: Grösse, Grenze, Argument und Fuuction" war das erste Ergebriss dieser mühevollen Bestrebungen. Dem ersten Theil, der auch in's Französische übersetzt wurde, ist ein zweiter Theil nicht mehr gefolgt. In diesem Werk wendet er sich gegen die rein formale Auffassung des Zahlbegriffs; nur unter der Voraussetzung eines gezählten oder gt:messenen Objectes hat ihm die Zah! eine Bedeutung, und so kommt er zu dem Schluss, dass zwei grundyerschiedepe Auffassungen mäglich und berechtigt sind, die er die idealistische und die empiristische nennt, deren jede in ihrer Weise zum Ziele kommt. Die Darstellung dieser entgegengesetzten Standpunkte kleidet er in die belebende Form einer Controverse zwischen dem Idealistep und Empiristen, von denen der erste seinen Grundsaty, den Glauben an die wirkliche Existen\% des uneudlich Grossen und unendlich Kleinen, der andere seiner entgegengesetzten Standpunkt und seinen Widerspruch gegen die Annabme genaner Maasse berründet. Zu einem Austrag wird der Streit nicht gebracht, sondern beide Systeme gehen neben einander ber und bestehen mit einander. Es ist aber hier nicht der Ort nod in der Kürze nicht möglich, auf das Hinzelne dieser originellen Betrachtungsaud Darstellungsweise und das Für und Wider einzugehen.

In der letzten Zeit seines Lebens bat du Bois-Reymond diesen 
Speculationen, bei denen, wie er oft aussprach, die Ergebnisse in allzu ungünstigem Verhältnisse zu der aufgewandten Zeit und Arbeit stehen, sich abgewandt und ist, getreu seinen ersteu Interessen, zu den Arbeiten seiner jüngeren Jahre, den partiellen Differentialgleichungen, zurückgekehrt. Die letzte kurz vor seinem Tode im Journal für Mathematik erschienene Arbeit „Ueber lineare partielle Differentialgleichungen zweiter Ordnung" knüpft anmittelbar an sein erstes grösseres Werk, die "Beiträge" an und sollte der Ausgangspunkt für eine Reihe von weiteren Forschungen sein, von denen wir uns noch mauchen Aufschluss hätten versprechen dürfen.

Ueber die Lebensschicksale du Bois-Reymonds ist wenig noch nachzutragen. In Jahre 1870 wurde er als ordentlicher Professor an die Universität Freiburg berufen, wo er bis 1874 wirkte. In diesem Jahre folgte er einem Rufe nach Tübingen als Nachfolger H. Hankel's. Hier hat er sich verheirathet und mehrere Jahre einer erfolgreichen Lehrthätigkeit verlebt. Die engen VerhäItnisse einer kleinen Universität waren aber seinem Wesen nicht angemessen; die Liebe zu seiner Geburtsstadt Berlin hat ihn nie verlassen, und so war es ihm die Erfüllang eines Herzenswunsches, als er 1884 als Professor der Mathematik an die Berliner technische Hochschule berufen wurde, welche gerade damals ihren neuen Palast in Charlottenburg bezog. Lange durfte er sich der neuen Thätigkeit nicht erfreuen. Am 7. April 1889 ist er auf der Durchreise nach Neuchatel in Freiburg einem Nierenleiden erlegen, unter dem er die letaten Jahre seines Lebens schwer zu leiden hatte.

Es konnte nicht die Absicht dieser Blätter sein, eine auch nur einigermassen erschöpfende Darstellung der wissenschaftlichen Arbeiten und Leistungen des Verstorbenen zu geben. Nur Einzelnes haben wir hervorgehoben, was für seine Richtung und seinen wissenschaftlichen Standpunkt uns bezeichnend zu sein schien. Unsere Aufgabe war, dem Leser ein Bild des Lebens und Schaffens eines Mannes vorzuführen, dessen höchstes Streben die Wissenschaft war, deren Zielen er mit rastlosem Eifer nachtrachtete, bis der Tod ihm die Feder aus der Hand nahm.

Marburg, im December 1889.

H. Weber. 